TRANSACTIONS OF THE

AMERICAN MATHEMATICAL SOCIETY

Volume 352, Number 9 , Pages 4007-4017

S 0002-9947(00)02524-1

Article electronically published on March 15, 2000

\title{
DIHEDRAL COVERINGS OF ALGEBRAIC SURFACES AND THEIR APPLICATION
}

\author{
HIRO-O TOKUNAGA
}

\begin{abstract}
In this article, we study dihedral coverings of algebraic surfaces branched along curves with at most simple singularities. A criterion for a reduced curve to be the branch locus of some dihedral covering is given. As an application we have the following:

Let $B$ be a reduced plane curve of even degree $d$ having only $a$ nodes and $b$ cusps. If $2 a+6 b>2 d^{2}-6 d+6$, then $\pi_{1}\left(\mathbf{P}^{2} \backslash B\right)$ is non-abelian.

Note that Nori's result implies that $\pi_{1}\left(\mathbf{P}^{2} \backslash B\right)$ is abelian, provided that $2 a+6 b<d^{2}$.
\end{abstract}

\section{INTRODUCTION}

Let $\Sigma$ be a smooth surface, and $S$ a normal surface equipped with a finite morphism $\pi: S \rightarrow \Sigma$. We call $S$ a $\mathcal{D}_{2 n}$ covering if the rational function field, $\mathbf{C}(S)$, of $S$ is a Galois extension of $\mathbf{C}(\Sigma)$ with Galois group $\mathcal{D}_{2 n}$ : the dihedral group of order $2 n$. In previous articles [T1, T2 and [T4], we developed a general theory for $\mathcal{D}_{2 n}$ coverings and studied such coverings of $\mathbf{P}^{2}$. In this article, we continue to study the problem of existence of a $\mathcal{D}_{2 n}$ covering possessing a given branch locus. More precisely, we consider the following:

Question 0.1. (a) Let $B$ be a reduced divisor on $\Sigma$ with at most simple singularities. Give a condition for $B$ to be the branch locus of some $\mathcal{D}_{2 n}$ covering $\pi: S \rightarrow \Sigma$ which is branched along $B$. Here simple singularities of curves are those in II, $\S 8$, BPV].

(b) ( $A$ weak version of $(a)$.) Let $B$ be as above. Give a condition for $B$ to be the branch locus of some $\mathcal{D}_{2 n}$ covering $\pi: S \rightarrow \Sigma$ which is branched along $B$ with ramification index 2 . Here, the ramification index means one along the smooth part of $B$.

Note that, if $\Sigma$ is simply connected and $B$ is irreducible, then any $\mathcal{D}_{2 n}$ covering is branched along $B$ with ramification index 2 .

In [T1 and T2, we studied Question 0.1 (a) in the case where $\Sigma=\mathbf{P}^{2}$ and $\operatorname{deg} B=4,5$; and gave a characterization of possible branch curves in terms of the combination of singularities. In [T4, we considered the same question in the case where $\Sigma=\mathbf{P}^{2}$ and $B$ is an irreducible maximizing sextic curve with a triple point. In this article, we consider Question 0.1 (b) under more general settings as follows:

Received by the editors June 20, 1998.

2000 Mathematics Subject Classification. Primary 14E20; Secondary 14E15.

This research is partly supported by the Grant-in-Aid for Encouragement of Young Scientists 09740031 from the Ministry of Education, Science and Culture.

(C)2000 American Mathematical Society 
As we will see in $\S 1$, if the $\mathcal{D}_{2 n}$ covering in Question 0.1 (b) exists, then there exists a finite double covering $f^{\prime}: Z^{\prime} \rightarrow \Sigma$ branched along $B$. This shows that as far as we consider Question 0.1(b), there is no harm to assume the existence of $Z^{\prime}$. We denote its canonical resolution by $Z$. By the definition of the canonical resolution (see $[\mathrm{H}]$ ), $Z$ satisfies the following diagram:

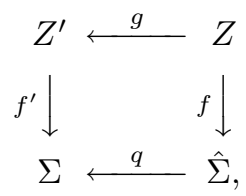

where $q: \hat{\Sigma} \rightarrow \Sigma$ is a composition of blowing-ups so that the induced morphism $f$ is finite. We also denote the composite morphism $q \circ f$ by $\tilde{f}$. Note that $Z^{\prime}$ is determined by $B$ and a line bundle $\mathcal{L}$ on $\Sigma$ such that $2 \mathcal{L} \sim B$ (see [BPV], I.17).

In this article, we study Question 0.1 (b) under the assumptions:

Assumption 0.2. The Néron-Severi group, $\mathrm{NS}(Z)$, of $Z$ is torsion free.

Let $T$ be a subgroup of $\operatorname{NS}(Z)$ generated by $\tilde{f}^{*} \mathrm{NS}(\Sigma)$ and all the irreducible components of the exceptional divisor of the resolution $g: Z \rightarrow Z^{\prime}$. Now we are in a position to state our results.

Theorem 0.3. Let $B$ be a curve as in Question 0.1(b) and suppose that $Z$ satisfies Assumption 0.2. Let $n$ be a fixed positive odd integer. If $\mathrm{NS}(Z) / T$ has a torsion of order $n$ and $\operatorname{gcd}\left(n, \operatorname{disc}\left(\tilde{f}^{*} \mathrm{NS}(\Sigma)\right)\right)=1$, where $\operatorname{disc}\left(\tilde{f}^{*} \mathrm{NS}(\Sigma)\right)$ is the determinant of the intersection matrix of the lattice $\tilde{f}^{*} \mathrm{NS}(\Sigma)$, then there exists a $\mathcal{D}_{2 n}$ covering of $\Sigma$ branched along $B$ with ramification index 2 .

Using Theorem 0.3 , we give a criterion for the existence of a $\mathcal{D}_{2 p}$ ( $p$ : odd prime) covering in terms of the number of singularities. To state our result, we need to define the total Milnor number (or the index) of $B$. For a singularity $x \in \operatorname{Sing}(B)$, we denote its Milnor number by $\mu_{x}$. For simple singularities, $\mu_{x}=$ the subindex of the type of $x$, where we use the notation $a_{n}, d_{n}$ and $e_{n}$ to describe the types of simple singularities. The total Milnor number, $\mu(B)$, of $B$ is $\mu(B)=\sum_{x \in \operatorname{Sing}(B)} \mu_{x}$.

Theorem 0.4. Let $B$ be a curve as in Question 0.1(b) and suppose that $Z$ satisfies Assumption 0.2. Let $\mathcal{L}$ be the line bundle as above. Let $p$ be a fixed odd prime. Define a non-negative integer $l$ to be

$l=$ the number of singularities of type $a_{3 k-1}(k>0)$ and $e_{6}$, if $p=3$, and;

$l=$ the number of singularities of type $a_{p k-1}(k>0)$, if $p \geq 5$.

If $p$ Xdisc $\left(\tilde{f}^{*} \mathrm{NS}(\Sigma)\right)$ and

$$
\begin{aligned}
l>24 & \left(\mathcal{O}_{\Sigma}\right)+4\left(h^{1}\left(\mathcal{O}_{\Sigma}\right)+h^{1}\left(\mathcal{O}_{\Sigma}(-\mathcal{L})\right)\right) \\
& +4 \mathcal{L}^{2}+2 K_{\Sigma} \mathcal{L}-2 K_{\Sigma}^{2}-2-\rho(\Sigma)-\mu(B),
\end{aligned}
$$

where $K_{\Sigma}$ is the canonical bundle of $\Sigma$, then there exists a $\mathcal{D}_{2 p}$ covering of $\Sigma$ branched along $B$ with ramification index 2 .

Note that Theorems 0.3 and 0.4 are generalization of the corresponding results (Theorem 0.6 and Proposition 3.1) in [T4. 
We apply Theorem 0.3 to studying the fundamental group of the complement to the branch curve. It is easy to see that, if a $\mathcal{D}_{2 n}$ covering $\pi: S \rightarrow \Sigma$ with $B$ as the branch locus exists, then there exists a surjective homomorphism from $\pi_{1}(\Sigma \backslash B) \rightarrow \mathcal{D}_{2 n}$. This implies that $\pi_{1}(\Sigma \backslash B)$ is non-abelian. In particular, as we will see in $\S 4$, Assumption 0.2 holds when $\Sigma=\mathbf{P}^{2}$. Hence we have the following corollaries.

Corollary 0.5. Let $p$ be a fixed odd prime. Let $B$ be a plane curve of degree $2 m$ with at most simple singularities. Let $l$ be the integer as in Theorem 0.4. If $l>4 m^{2}-6 m+3-\mu$, then there exists a $\mathcal{D}_{2 p}$ covering, $S$, of $\mathbf{P}^{2}$ branched along $B$ with ramification index 2 . In particular, $\pi_{1}\left(\mathbf{P}^{2} \backslash B\right)$ is non-abelian.

In particular, if $B$ has only nodes and cusps, we have the following:

Corollary 0.6. Suppose that $B$ has a nodes and $b$ cusps. If $2 a+6 b>2(2 m)^{2}-$ $6(2 m)+6$, then there exists a $\mathcal{D}_{6}$ covering, $S$, of $\mathbf{P}^{2}$ branched along $B$ with ramification index 2 . In particular, $\pi_{1}\left(\mathbf{P}^{2} \backslash B\right)$ is non-abelian.

Remark 0.7. (i) The inequality in Corollary 0.5 is sharp in the following sense:

There exist pairs of irreducible plane sextic curves $\left(B_{1}, B_{2}\right)$ such that

(a) both $B_{1}$ and $B_{2}$ have the same combination of singularities; and it is either $3 e_{6}$ or $e_{6}, 4 a_{2}, 2 a_{1}$, and

(b) there exists a $D_{6}$ covering branched along $B_{1}$ with ramification index 2 , while there is no such covering for $B_{2}$.

For details, see [A], [T3] and [T5].

(ii) By [No], it is known that, if $2 a+6 b<(2 m)^{2}$, then $\pi_{1}\left(\mathbf{P}^{2} \backslash B\right)$ is abelian. This contrast makes our result more interesting.

(iii) In [Q], Oka recently showed that there exist sextic curves, $B_{1}$ and $B_{2}$, with 3 nodes and 6 cusps such that $\pi_{1}\left(\mathbf{P}^{2} \backslash B_{1}\right)$ is non-abelian, while $\pi_{1}\left(\mathbf{P}^{2} \backslash B_{2}\right)$ is abelian. This shows that the inequality Corollary 0.6 is the best possible.

\section{ACKNOWLEDGMENTS}

Part of this work was done during the author's visit to the Ruhr Universität, Bochum under the support of SFB 237. Deep appreciation goes to the university for its nice atmosphere, and above all, to Alan Huckleberry for his hospitality and arrangements. The author also expresses gratitude to M. Oka and I. Shimada for their comments on the relation between the results in this article and the fundamental group.

Notations and Conventions. In this paper, the ground field is always the complex number field $\mathbf{C}$.

Let $X$ be a normal variety, and let $Y$ be a smooth variety. Let $\pi: X \rightarrow Y$ be a finite morphism from $X$ to $Y$. We define the branch locus of $f$, which we denote by $\Delta(X / Y)$, as follows:

$$
\Delta(X / Y)=\left\{y \in Y \mid \sharp\left(\pi^{-1}(y)\right)<\operatorname{deg} \pi\right\} .
$$

Let $S$ be a finite double covering of a smooth projective surface $\Sigma$. The "canonical resolution" of $S$ always means the resolution given by Horikawa in [H].

For simple singularities of curves, we use the notations, $a, d$ and $e$, to describe the types of such singularities. Note that the capital letters, $A, D$ and $E$ are used for the corresponding types in $[\mathrm{BPV}]$. 
Let $D_{1}, D_{2}$ be divisors.

$D_{1} \sim D_{2}$ : linear equivalence of divisors.

$D_{1} \sim \sim_{\mathbf{Q}} D_{2}$ : Q-linear equivalence of divisors.

$D_{1} \approx D_{2}$ : algebraic equivalence of divisors.

$D_{1} \approx_{\mathbf{Q}} D_{2}$ : Q-algebraic equivalence of divisors.

\section{Preliminaries}

This section consists of 3 subsections. All are summaries on the known facts and results, which we need to prove Theorems 0.3 and 0.4 .

1. Summary on $\mathcal{D}_{2 n}$ ( $n$ : odd) coverings. In this article, we only consider the case that the Galois group is $\mathcal{D}_{2 n}$, ( $n$ : odd).

We denote by $\mathcal{D}_{2 n}$ a dihedral group of order $2 n$, which is generated by two elements $\sigma$ and $\tau$ with the relations $\sigma^{2}=\tau^{n}=(\sigma \tau)^{2}=1$.

Let $\pi: X \rightarrow Y$ be a $\mathcal{D}_{2 n}$ covering. Let $\mathbf{C}(X)^{\tau}$ be the invariant subfield of $\mathbf{C}(X)$ by $\tau$. $\mathbf{C}(X)^{\tau}$ is a quadratic extension of $\mathbf{C}(Y)$. Let $D(X / Y)$ be the $\mathbf{C}(X)^{\tau}$ normalization of $Y . D(X / Y)$ is a double covering of $Y$ satisfying a commutative diagram as follows:

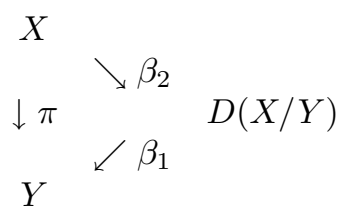

where $\beta_{1}: D(X / Y) \rightarrow Y$ is a double covering of $Y$ and $\beta_{2}: X \rightarrow D(X / Y)$ is an $n$-fold cyclic covering of $D(X / Y)$. Note that $\Delta(D(X / Y) / Y)=\Delta(X / Y)$ if $\pi$ is a $\mathcal{D}_{2 n}$ covering branched along $B$ with ramification index 2 . We fix these notations throughout this article. The following proposition is fundamental in constructing a $\mathcal{D}_{2 n}$ covering.

Proposition 1.1. Let $n$ be an odd integer $\geq 3$. Let $f: Z \rightarrow Y$ be a smooth finite double covering of $Y$. We denote the covering transformation by $\sigma$. Let $D$ be an effective divisor such that

(i) $D$ and $\sigma^{*} D$ have no common component,

(ii) if we let $D=\sum_{i} a_{i} D_{i}$ be the irreducible decomposition, then for all $i, a_{i}>0$ and the greatest common divisor of $a_{i}$ 's and $n$ is 1 , and

(iii) there exists a line bundle $L$ such that $D-\sigma^{*} D \approx n L$.

Then there exists a $\mathcal{D}_{2 n}$ covering, $X$, of $Y$ such that

(a) $D(X / Y)=Z$ and $(b)$ the branch locus of $\beta_{2}, \Delta(X / Z)$, is contained in $\operatorname{Supp}\left(D+\sigma^{*} D\right)$, i.e., $\Delta(X / Y) \subset \Delta(Z / Y) \cup f(\operatorname{Supp}(D))$.

Proof. It is enough to find the three effective divisors $D_{1}, D_{2}$ and $D_{3}$ on $Z$ satisfying the three conditions in Proposition 0.4 in [T1]. We first define $D_{1}$ in the following way:

For the coefficient of each irreducible component $D_{i}$ of $D$, put $a_{i}^{\prime}=a_{i}-n\left[\frac{a_{i}}{n}\right]$, where [] denotes the greatest integer function. As $n \nmid a_{i}, a_{i}^{\prime}>0$. We rewrite 
$D-\sigma^{*} D$ as follows:

$$
\begin{aligned}
D-\sigma^{*} D & =\sum_{i}\left(a_{i}^{\prime} D_{i}-a_{i}^{\prime} \sigma^{*} D_{i}+n\left[\frac{a_{i}}{n}\right]\left(D_{i}-\sigma^{*} D_{i}\right)\right) \\
& =\sum_{i, a_{i}^{\prime} \leq \frac{n-1}{2}}\left(a_{i}^{\prime} D_{i}-a_{i}^{\prime} \sigma^{*} D_{i}+n\left[\frac{a_{i}}{n}\right]\left(D_{i}-\sigma^{*} D_{i}\right)\right) \\
& +\sum_{i, a_{i}^{\prime}>\frac{n-1}{2}}\left(\left(n-a_{i}^{\prime}\right) \sigma^{*} D_{i}-\left(n-a_{i}^{\prime}\right) D_{i}+n\left(\left[\frac{a_{i}}{n}\right]+1\right)\left(D_{i}-\sigma^{*} D_{i}\right)\right) .
\end{aligned}
$$

Put

$$
D_{1}=\sum_{i, a_{i}^{\prime} \leq \frac{n-1}{2}} a_{i}^{\prime} D_{i}+\sum_{i, a_{i}^{\prime}>\frac{n-1}{2}}\left(n-a_{i}^{\prime}\right) \sigma^{*} D_{i} .
$$

(Note that the greatest common divisor of the coefficients, $a_{i}^{\prime}$ 's, and $n$ is 1 by the assumption (ii).) Since $\operatorname{Pic}^{0}(Z)$ is $n$-divisible, by replacing $L$ by a suitable one, we may assume that $D-\sigma^{*} D \sim n L$. Hence we have

$D_{1}-\sigma^{*} D_{1} \sim n\left(L-\sum_{i, a_{i}^{\prime} \leq \frac{n-1}{2}}\left[\frac{a_{i}}{n}\right]\left(D_{i}-\sigma^{*} D_{i}\right)-\sum_{i, a_{i}^{\prime}>\frac{n-1}{2}}\left(\left[\frac{a_{i}}{n}\right]+1\right)\left(D_{i}-\sigma^{*} D_{i}\right)\right)$.

Since any line bundle is linear equivalent to a difference of two effective divisors, the right hand side in the above is linear equivalent to a divisor of form $n\left(D_{3}-D_{2}\right)$ where $D_{2}$ and $D_{3}$ are effective divisors. Thus we have three effective divisors $D_{1}$, $D_{2}$ and $D_{3}$ satisfying the three conditions in Proposition 0.4.

2. Preliminary from lattice theory. Here we summarize some elementary facts from lattice theory.

Let $L$ be a lattice, i.e.,

(i) $L$ is a free finite $\mathbf{Z}$ module and

(ii) $L$ is equipped with a non-degenerate bilinear symmetric pairing $\langle$,$\rangle .$

For a given lattice $L$, disc $L$ is the determinant of the intersection matrix. Note that it is independent of choice of a basis. We call $L$ unimodular if $\operatorname{disc} L= \pm 1$.

Let $J$ be a sublattice of $L$. We denote its orthogonal complement with respect to $\langle$,$\rangle by J^{\perp}$.

For a lattice $L$, we denote its dual lattice by $L^{\vee}$. Note that, by using the pairing, $L$ is embedded in $L^{\vee}$ as a sublattice with same rank. Hence the quotient group $L^{\vee} / L$ is a finite abelian group, which we denote by $G_{L}$.

Although the following lemma is often used under the assumption that $L$ is even unimodular, the statement also holds for any unimodular lattice (see $[\mathrm{E}]$ ).

Lemma 1.2. Let $L$ be a unimodular lattice. Let $J_{1}$ and $J_{2}$ be sublattices of $L$ such that $J_{1}^{\perp}=J_{2}$ and $J_{2}^{\perp}=J_{1}$. Then

$$
G_{J_{1}} \cong G_{J_{2}} .
$$

A sublattice $M$ of $L$ is called primitive if $L / M$ is torsion-free.

Example 1.3. Let $S$ be a surface. Since $H^{2}(S, \mathbf{Z}) / \operatorname{NS}(S)$ is torsion free, $\operatorname{NS}(S)$ is torsion free if and only if $H^{2}(S, \mathbf{Z})$ is. Therefore for the surface $Z$ in Theorems 0.3 and $0.4, H^{2}(Z, \mathbf{Z})$ is torsion free. In fact, it is a unimodular lattice with respect to the intersection pairing, and $\mathrm{NS}(S)$ is a primitive sublattice of it. 
3. Analysis of the torsion part of $\mathrm{NS}(Z) / T$. We use the same notations as those in Introduction. Throughout this subsection, we always assume that $\operatorname{NS}(Z)$ is torsion free. We start with the following lemma.

Lemma 1.4. $(\mathrm{NS}(Z) / T)_{\text {tor }}=T^{\perp \perp} / T$.

Proof. By definition, $(\mathrm{NS}(Z) / T)_{\text {tor }} \subset T^{\perp \perp} / T$, and we have $T^{\perp \perp} \subset \mathrm{NS}(Z)$ by Example 1.3. This implies our lemma.

$T^{\perp \perp}$ is considered as a sublattice of $T^{\vee}$ by using the intersection pairing. By Lemma 1.4, this means that the torsion subgroup of $\mathrm{NS}(Z) / T$ is considered as a subgroup of $G_{T}$. We next look at $G_{T}$.

Let $x$ be a singular point of $B$. Put

$R_{x}=$ the subgroup of $T$ generated by all irreducible components of the exceptional divisor of $f^{-1}(x)$.

Then we have a decomposition of $T$ as follows:

$$
T \cong \tilde{f}^{*} \mathrm{NS}(\Sigma) \oplus \bigoplus_{x \in \operatorname{Sing}(B)} R_{x}
$$

With this decomposition, we have $T^{\vee} / T \cong G_{\tilde{f}^{*} \mathrm{NS}(\Sigma)} \oplus \bigoplus_{x \in \operatorname{Sing}(B)} G_{R_{x}}$. Note that, for each $G_{R_{x}}$, we have the following classical fact:

Fact 1.5. Since we only consider a torsion element of odd order, the cases of $a_{n}$ and $e_{6}$ are all we need. We consider both $R_{x}$ and $R_{x}^{\vee}$ as subgroups of $R_{x} \otimes \mathbf{Q}$ and give a $\mathbf{Q}$-divisor that gives rise to a generator of $G_{R_{x}}$. To this purpose, we label irreducible components of the exceptional divisors for $A_{n}$ and $E_{6}$ singularities (see Figure 1).

\begin{tabular}{|c|c|c|}
\hline The type of $x$ & The type of $f^{\prime-1}(x)$ & $G_{R_{x}}$ \\
\hline$a_{n}$ & $A_{n}$ & $\mathbf{Z} /(n+1) \mathbf{Z}$ \\
\hline$d_{n}, n \equiv 1 \bmod 2$ & $D_{n}$ & $\mathbf{Z} / 4 \mathbf{Z}$ \\
\hline$d_{n}, n \equiv 0 \bmod 2$ & $D_{n}$ & $(\mathbf{Z} / 2 \mathbf{Z})^{\oplus 2}$ \\
\hline$e_{6}$ & $E_{6}$ & $\mathbf{Z} / 3 \mathbf{Z}$ \\
\hline$e_{7}$ & $E_{7}$ & $\mathbf{Z} / 2 \mathbf{Z}$ \\
\hline$e_{8}$ & $E_{8}$ & $\{0\}$ \\
\hline
\end{tabular}

Lemma 1.6. $G_{R_{x}}$ is generated by the class of $\mathbf{Q}$-divisors $D_{x} /(b+1)\left(\right.$ resp. $\left.\frac{D_{x}}{3}\right)$ for $x=a_{b}\left(\right.$ resp. $\left.x=e_{6}\right)$, where

$$
D_{x}=\left\{\begin{array}{l}
\sum_{k=1}^{\frac{b}{2}}(b+1-k)\left(\Theta_{k}-\Theta_{b-k}\right), \quad \text { if } x=a_{b} b: \text { even, } \\
\sum_{k=1}^{\frac{b-1}{2}}(b+1-k)\left(\Theta_{k}-\Theta_{b-k}\right)+\frac{b+1}{2} \Theta_{\frac{b+1}{2}} \quad \text { if } x=a_{b} b: \text { odd },
\end{array}\right.
$$

and

$$
D_{x}=\left(\Theta_{1}-\Theta_{5}\right)+2\left(\Theta_{2}-\Theta_{6}\right) \text { if } x=e_{6} .
$$

Proof. The inverse of the intersection matrix of $R_{x}$ shows that a generator is given by the class of $\mathbf{Q}$-divisors

$$
\frac{1}{b+1}\left(b \Theta_{1}+(b-1) \Theta_{2}+\cdots+\Theta_{b}\right) \quad \text { for } x=a_{b},
$$




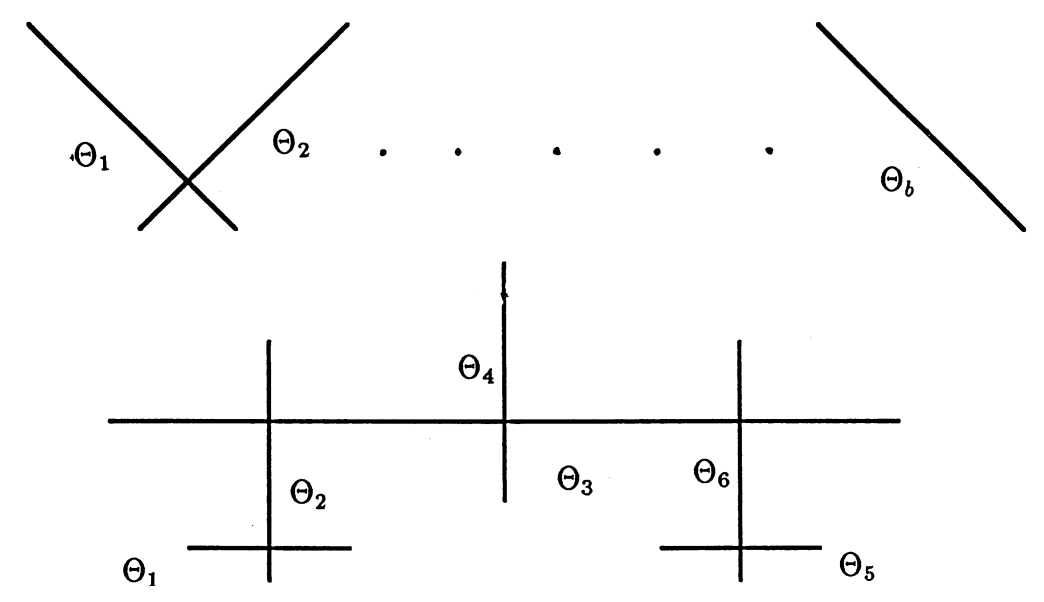

FiguRE 1.

and

$$
\frac{1}{3}\left(4 \Theta_{1}+5 \Theta_{2}+6 \Theta_{3}+3 \Theta_{4}+2 \Theta_{5}+4 \Theta_{6}\right) \quad \text { for } x=e_{6} .
$$

Our statement is straightforward from these $\mathbf{Q}$-divisors.

Remark 1.7. Let $\sigma$ denote the covering transformation of the double covering $f$ : $Z \rightarrow \hat{\Sigma}$. Then we have $\sigma^{*} \Theta_{k}=\Theta_{n-k}\left(1 \leq k \leq\left[\frac{n}{2}\right]\right)$ for the exceptional divisor of an $A_{n}$ singularity and $\sigma^{*} \Theta_{1}=\Theta_{5}$ and $\sigma^{*} \Theta_{2}=\Theta_{6}$ for those of an $E_{6}$ singularity by the construction of the canonical resolution.

\section{Proof of Theorem 0.3}

We first reduce the existence of a dihedral covering of $\Sigma$ to that of $\hat{\Sigma}$. Suppose that there exists a $\mathcal{D}_{2 n}$ covering, $\hat{S}$, of $\hat{\Sigma}$ such that

(i) $D(\hat{S} / \hat{\Sigma})=Z$, and

(ii) $\Delta(\hat{S} / Z) \subset$ the support of the exceptional divisors of $g: Z \rightarrow Z^{\prime}$.

Then the Stein factorization, $S$, of $q \circ \hat{\pi}: \hat{S} \rightarrow \Sigma$ gives rise to the desired $\mathcal{D}_{2 n}$ covering. Hence by Proposition 1.1 we only need to prove the following:

Proposition 2.1. There exist a divisor $D$ and a line bundle $L$ such that

(i) $\operatorname{Supp}\left(D+\sigma^{*} D\right) \subset \operatorname{Supp}($ the exceptional divisor of $g)$, and

(ii) the pair $(D, L)$ satisfies the condition in Proposition 1.1.

We need several steps to prove Proposition 2.1.

Let $\nu$ be the homomorphism $T^{\perp \perp} \rightarrow T^{\vee} \rightarrow G_{T}$. Let $L_{1}$ be an element of $T^{\perp \perp}$ which gives rise to an $n$-torsion in $T^{\perp \perp} / T$. As $G_{T} \cong G_{\tilde{f}^{*} \mathrm{NS}(\Sigma)} \oplus \bigoplus_{x \in \operatorname{Sing}(B)} G_{R_{x}}$, we write

$$
\nu\left(L_{1}\right)=\left(\alpha,\left(\beta_{x}\right)_{x \in \operatorname{Sing}(B)}\right) \in G_{\tilde{f}^{*} \mathrm{NS}(\Sigma)} \oplus \bigoplus_{x \in \operatorname{Sing}(B)} G_{R_{x}} .
$$

Lemma 2.2. $\alpha=0$.

Proof. As $\sharp G_{\tilde{f}^{*} \mathrm{NS}(\Sigma)}=\operatorname{disc} \tilde{f}^{*} \mathrm{NS}(\Sigma), \alpha=0$ by the assumption.

Lemma 2.3. If $x$ is neither $a_{n}$ nor $e_{6}, \beta_{x}=0$. 
Proof. This is immediate by Fact 1.5.

For simplicity, we put $r_{x}=\sharp\left(G_{R_{x}}\right)$ in the following. Note that $r_{x}=b+1$ (resp. 3 ) if $x$ is of type $a_{b}$ (resp. $e_{6}$ ).

Lemma 2.4. Suppose $\beta_{x} \neq 0$. Let $s_{x}$ be the order of $\beta_{x}$. As $s_{x}$ is a common divisor of $n$ and $s_{x}$, we put $r_{x}=s_{x} t_{x}$ and $n=s_{x} u_{x}$. Then there exists an integer $k_{x}, 0<k_{x}<s_{x},\left(k_{x}, s_{x}\right)=1$ such that

$$
\beta_{x}=\text { the class of } \frac{k_{x}}{s_{x}} D_{x},
$$

where $D_{x}$ is the divisor in Lemma 1.6.

Proof. As $\beta_{x} \neq 0, x$ is of either type $a_{n}$ or $e_{6}$. In such a case, $G_{R_{x}}$ is a cyclic group with a generator as in Lemma 1.6, from which our lemma follows.

By Lemma 2.4, we have

$$
L_{1} \approx_{\mathbf{Q}} \sum_{x \in \operatorname{Sing}(B)} \frac{k_{x}}{s_{x}} D_{x} \bmod T
$$

This means that there exists an element, $L_{2}$, in $T$ such that

$$
L_{1}+L_{2} \approx_{\mathbf{Q}} \sum_{x \in \operatorname{Sing}(B)} \frac{k_{x}}{s_{x}} D_{x} .
$$

Lemma 2.5. The greatest common divisor of the $\left(n k_{x} / s_{x}\right)$ 's is 1 .

Proof. Let $d=\operatorname{gcd}\left(\left(\frac{n k_{x}}{s_{x}}\right)_{x \in \operatorname{Sing} B}\right)$. Then $\frac{n}{d}\left(L_{1}+L_{2}\right) \in T$ as $\operatorname{NS}(Z)$ is torsion free. This shows that the order of $\nu\left(L_{1}+L_{2}\right)=\nu\left(L_{1}\right)$ is a divisor of $\frac{n}{d}$. By our assumption, the order of $\nu\left(L_{1}\right)$ is $n$; and $d=1$.

Now we define the divisor $D$ on $Z$ as follows:

If $\beta_{x} \neq 0, x=a_{b}, b$ : even, then put

$$
D_{x}^{+}=\frac{n k_{x}}{s_{x}} \sum_{k=1}^{\frac{b}{2}}(b+1-k) \Theta_{k} .
$$

If $\beta_{x} \neq 0, x=a_{b}, b$ : odd, then put

$$
D_{x}^{+}=\frac{n k_{x}}{s_{x}} \sum_{k=1}^{\frac{b-1}{2}}(b+1-k) \Theta_{k} .
$$

If $\beta_{x} \neq 0, x=e_{6}$, then put

$$
D_{x}^{+}=\frac{n k_{x}}{3} \Theta_{1}+\frac{2 n k_{x}}{3} \Theta_{2} .
$$

Now put $D=\sum_{x \in \operatorname{Sing}(B)} D_{x}^{+}$. Then

$$
D-\sigma^{*} D \approx n\left(L_{1}+L_{2}-\sum_{x=a_{b}, b: o d d, \beta_{x} \neq 0} \frac{k_{x} r_{x}}{2 s_{x}} \Theta_{\frac{r_{x}}{2}}\right) .
$$

Put $L=L_{1}+L_{2}-\sum_{x=a_{b}, b: o d d, \beta_{x} \neq 0} \frac{k_{x} r_{x}}{2 s_{x}} \Theta_{\frac{r_{x}}{2}}$. Then the pair $(D, L)$ satisfies the condition in Proposition 2.1. 


\section{Proof of Theorem 0.4 .}

Thanks to Theorem 0.3 , it is enough to show that $\mathrm{NS}(Z) / T$ has a $p$-torsion. We first prove the following lemma:

Lemma 3.1. Let $b_{i}(Z)$ be the $i$-th Betti number of $Z$. Then we have

$$
b_{2}(Z)=24 \chi\left(\mathcal{O}_{\Sigma}\right)+4\left(h^{1}\left(\mathcal{O}_{\Sigma}\right)+h^{1}\left(\mathcal{O}_{\Sigma}(-\mathcal{L})\right)\right)+4 \mathcal{L}^{2}+2 K_{\Sigma} \mathcal{L}-2 K_{\Sigma}^{2}-2 .
$$

Proof. By [H], Lemma 6 and its proof, we have

$$
\begin{aligned}
\chi\left(\mathcal{O}_{Z}\right) & =\frac{1}{2} \mathcal{L}\left(K_{\Sigma}+\mathcal{L}\right)+2 \chi\left(\mathcal{O}_{\Sigma}\right), \\
K_{Z}^{2} & =2\left(K_{\Sigma}+\mathcal{L}\right)^{2} \quad \text { and }, \\
h^{1}\left(\mathcal{O}_{Z}\right) & =h^{1}\left(\mathcal{O}_{Z}\right)+h^{1}\left(\mathcal{O}_{Z}(-\mathcal{L})\right) .
\end{aligned}
$$

Hence the Noether formula gives

$$
c_{2}(Z)=12 \chi\left(\mathcal{O}_{Z}\right)-c_{1}^{2}(Z)=24 \chi\left(\mathcal{O}_{Z}\right)+4 \mathcal{L}^{2}+2 K_{\Sigma} \mathcal{L}-2 K_{\Sigma}^{2} .
$$

As $b_{1}(Z)=b_{3}(Z)=2 h^{1}\left(\mathcal{O}_{Z}\right)$, we have the desired equality.

In the following, we make use of some Nikulin theory $([\mathrm{N}])$. This approach is a modification of Miranda-Persson's in $\S 4$, $[\mathrm{MP}$. A similar argument is also found in X].

Suppose that there exists no $p$-torsion in $T^{\perp \perp} / T$. Then

$$
S_{p}\left(G_{T}\right) \cong S_{p}\left(G_{T^{\perp \perp}}\right),
$$

where $S_{p}(G)$ denotes the $p$-Sylow group of $G$. By Lemma 1.6 and Example 1.7, we have

$$
G_{T^{\perp}} \cong G_{T^{\perp \perp}} .
$$

Hence the number of generators, $l_{1}$, of $S_{p}\left(G_{T^{\perp \perp}}\right) \leq \operatorname{rank} T^{\perp}=b_{2}(Z)-\operatorname{rank} T$. On the other hand, by the assumption and Lemma 3.1, we have $l_{1} \geq l>b_{2}(Z)-\operatorname{rank} T$. This leads us to a contradiction.

\section{Applications}

We keep the same notations as before.

Application 1: $\mathcal{D}_{2 n}$ coverings of $\mathbf{P}^{2}$.

Lemma 4.1. Suppose that

(i) $\Sigma$ is simply connected, and

(ii) the linear system $|B|$ is a base point and fixed component free.

Then $\pi_{1}(Z)=0$. In particular, $\mathrm{NS}(Z)$ is torsion free.

Proof. Let $B_{1}$ be a smooth member of $|B|$. Then there exists a smooth double covering $Z_{1}$ of $\Sigma$ branched along $B_{1}$. By Brieskorn's results for the simultaneous resolution of rational double points (B1], B2]), $Z$ is considered as a smooth deformation of $Z_{1}$. This implies that $Z$ is homeomorphic to $Z_{1}$. By Proposition 1.8 , C], $Z_{1}$ is simply connected. Hence $Z$ is simply connected.

In the case where $\Sigma=\mathbf{P}^{2}$, the two assumptions in Lemma 4.1 always hold. This gives Corollary 0.5 
Example 4.2. (i) Put $p=3$ and $m=2$. Consider a plane quartic curve having $3 a_{2}$ singularities. Then there exists a $\mathcal{D}_{6}$ covering branched along $B$ with the ramification index 2. This gives another proof for Zariski's result: $\pi_{1}\left(\mathbf{P}^{2} \backslash B\right)$ is non-abelian ( $\mathrm{Z} \mid)$.

(ii) Put $p=3$ and $m=3$. Let $B$ be the sextic curve as in the table in $\S 6$, [T4]. Then there exists a $\mathcal{D}_{6}$ covering branched along $B$ with the ramification index 2 . Note that we proved the existence for only the first 7 cases in [T4].

Remark 4.3. The author does not know any single example of a plane curve of degree $\geq 8$ enjoying the assumption in Corollary 0.5. Also, he does not know of any single example of a plane curve enjoying the assumption in Corollary 0.5 for $p \geq 5$.

Application 2: $n$-torsions of an elliptic surface and $\mathcal{D}_{2 n}$ coverings

In [T1], T2] and [T4], $p$-torsions of the Mordell-Weil group of an elliptic surface play important roles in constructing $\mathcal{D}_{2 p}$ ( $p$ : odd prime) coverings. We show that the results on the existence of $\mathcal{D}_{2 p}$ coverings in previous articles follow easily from Theorem 0.4.

Let $\varphi: \mathcal{E} \rightarrow C$ be an elliptic surface over a curve $C$ such that

(i) $\varphi$ is relatively minimal,

(ii) $\varphi$ has a section $s_{0}$, and

(iii) $\varphi$ has at least one singular fiber.

Under these assumptions, by Theorem 1.2 in $[\mathrm{S}], \mathrm{NS}(\mathcal{E})$ is torsion free. Also it is well-known that $\mathcal{E}$ is obtained as the canonical resolution of a double covering $f^{\prime}: \mathcal{E}^{\prime} \rightarrow \Sigma$ of some ruled surface $\Sigma$ over $C$ having the branch locus $\Delta\left(\mathcal{E}^{\prime} / \Sigma\right)$ in the form of $\Delta_{0}+B_{0}$ such that

(i) $\Delta_{0}$ is a section of $\Sigma$ and $B_{0}$ is a tri-section with $B_{0} \cap \Delta_{0}=\emptyset$,

(ii) the image of $s_{0}$ in $\Sigma$ is $\Delta_{0}$, and

(iii) $B_{0}$ has at most simple singularities.

We denote the morphism from $\mathcal{E} \rightarrow \Sigma$ by $\tilde{f}$.

We apply Theorem 0.3 to $\Sigma$ and $\Delta\left(\mathcal{E}^{\prime} / \Sigma\right)$ and obtain the following theorem, which is again a generalization of Proposition 3.1 in [T4].

Theorem 4.4. Let $n$ be an odd number. Let $M W(\mathcal{E})$ be the Mordell-Weil group of $\mathcal{E}$, i.e., the group of sections. If $M W(\mathcal{E})$ has an n-torsion, then there exists a $\mathcal{D}_{2 n}$ covering, $S$, of $\Sigma$ branched along $\Delta\left(\mathcal{E}^{\prime} / \Sigma\right)$ with ramification index 2.

Proof. As disc $\tilde{f}^{*} \operatorname{NS}(\Sigma)=4, \operatorname{gcd}\left(n, \operatorname{disc} \tilde{f}^{*} \operatorname{NS}(\Sigma)\right)=1$. Let $T$ be as before and let $T_{\varphi}$ be the subgroup of $\mathrm{NS}(\mathcal{E})$ generated by $s_{0}, F$ : a fiber of $\varphi$, and all the irreducible components of singular fibers not meeting $s_{0}$. By our construction, the difference between $T$ and $T_{\varphi}$ is just one between $\tilde{f}^{*} \mathrm{NS}(\Sigma)$ and $\mathbf{Z} s_{0} \oplus \mathbf{Z F}$. As $\tilde{f}^{*} \Delta_{0}=2 s_{0}$ and a fiber of $\Sigma \rightarrow C$ is also a fiber of $\varphi, \tilde{f}^{*} \mathrm{NS}(\Sigma)$ is a subgroup of $\mathbf{Z} s_{0} \oplus \mathbf{Z} F$ of index 2. This means that $\left[T_{\varphi}: T\right]=2$; and we have the exact sequence

$$
0 \rightarrow \mathbf{Z} / 2 \mathbf{Z} \rightarrow \mathrm{NS}(\mathcal{E}) / T \rightarrow \mathrm{NS}(\mathcal{E}) / T_{\varphi} \rightarrow 0
$$

This implies that, for $n$ : odd, $\mathrm{NS}(\mathcal{E}) / T$ has an $n$-torsion if and only if $\mathrm{NS}(\mathcal{E}) / T_{\varphi}$ does also. On the other hand, by Theorem 1.3 in $\left[\mathrm{S}, \operatorname{NS}(\mathcal{E}) / T_{\varphi} \cong M W(\mathcal{E})\right.$. By Theorem 0.3 , we have our statement.

\section{REFERENCES}

[A] E. Artal Bartolo: Sur les couples de Zariski, J. Alg. Geom. 3 (1994), 223-247. MR 94m:14033 
[BPV] W. Barth, C. Peters, and A. Van de Ven: Compact complex surfaces, Springer-Verlag, 1984. MR 86c:32026]

[B1] E. Brieskorn: Über die Auflösung gewisser Singularitäten von holomorpher Abbildungen, Math. Ann. 166, 76-102 (1966). [MR 34:6789

[B2] E. Brieskorn: Die Auflösung der rationalen Singuraritäten holomorpher Abbildungen, Math. Ann. 178, 255-270 (1968). MR 38:2140

[C] F. Catanese: On the moduli spaces of surfaces of general type, J. Diff. Geom. 19, 483-515 (1984). MR 86h:14031

[E] W. Ebeling: Lattices and Codes, Advanced Lectures in Math., Vieweg, 1994. MR 95c:11084

$[\mathrm{H}]$ E. Horikawa: On deformation of quintic surfaces, Invent. Math. 31, 43-85 (1975). MR 48:8511

[MP] R. Miranda and U. Persson: Configurations of $I_{n}$ fibers on elliptic K3 surfaces, Math. Z. 201, 339-361 (1989). MR 90h:14051

[Ni] V.V. Nikulin: Integral symmetric bilinear forms and some of their applications, Math. USSR Izv. 14 103-167 (1980).

[No] M. V. Nori: Zariski's conjecture and related problems, Ann. Sci. École Norm. Sup. 16, 305-344 (1983). MR 86d:14027

[O] M. Oka: Geometry of cuspidal sextics and their dual curves, preprint.

[S] T. Shioda: On the Mordell-Weil lattices, Comment. Math. Univ. St. Paul 39, 211-240 (1990). MR 91m:14056

[T1] H. Tokunaga: On dihedral Galois coverings, Canadian J. of Math., 46, 1299-1317 (1994). MR 94d:14015

[T2] H. Tokunaga: Dihedral coverings of $\mathbf{P}^{2}$ branched along quintic curves, preprint.

[T3] H. Tokunaga: A remark on E. Artal-Bartolo's paper: "On Zariski pairs", Kodai Math. J., 19, 207-217 (1996). MR 97f:14029

[T4] H. Tokunaga: Dihedral coverings branched along maximizing sextics, Math. Ann. 308, 633-648 (1997). MR 99d:14021

[T5] H. Tokunaga: Some examples of Zariski pairs arising from certain elliptic K3 surfaces, Math. Z. 227 (1998), 465-477. MR 99a:14055

[X] G. Xiao: Galois covers between K3 surfaces, Ann. Inst. Fourier 46, 73-88 (1996). MR 97b:14047

[Z] O. Zariski: On the problem of existence of algebraic functions of two variables possessing a given branch curves, Amer. J. Math. 51, 305-328 (1929).

Department of Mathematics and Information Science, Kochi University, Kochi 7808520, JAPAN

Current address: Department of Mathematics, Tokyo Metropolitan University, Minami-Osawa, Hachioji, Tokyo 192-0397 Japan

E-mail address: tokunagamath.kochi-u.ac.jp 